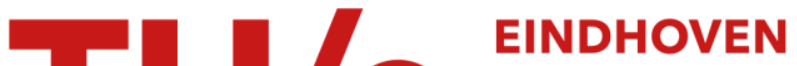 UNIVERSITY OF TECHNOLOGY
}

\section{On packing, connectivity, and conductivity in mesoscale networks of polydisperse multiwalled carbon nanotubes}

Citation for published version (APA):

Gnanasekaran, K., With, de, G., \& Friedrich, H. (2014). On packing, connectivity, and conductivity in mesoscale networks of polydisperse multiwalled carbon nanotubes. Journal of Physical Chemistry C, 118(51), 29796-

29803. https://doi.org/10.1021/jp5081669

DOI:

10.1021/jp5081669

Document status and date:

Published: 01/01/2014

\section{Document Version:}

Publisher's PDF, also known as Version of Record (includes final page, issue and volume numbers)

\section{Please check the document version of this publication:}

- A submitted manuscript is the version of the article upon submission and before peer-review. There can be important differences between the submitted version and the official published version of record. People interested in the research are advised to contact the author for the final version of the publication, or visit the $\mathrm{DOI}$ to the publisher's website.

- The final author version and the galley proof are versions of the publication after peer review.

- The final published version features the final layout of the paper including the volume, issue and page numbers.

Link to publication

\section{General rights}

Copyright and moral rights for the publications made accessible in the public portal are retained by the authors and/or other copyright owners and it is a condition of accessing publications that users recognise and abide by the legal requirements associated with these rights.

- Users may download and print one copy of any publication from the public portal for the purpose of private study or research.

- You may not further distribute the material or use it for any profit-making activity or commercial gain

- You may freely distribute the URL identifying the publication in the public portal.

If the publication is distributed under the terms of Article 25fa of the Dutch Copyright Act, indicated by the "Taverne" license above, please follow below link for the End User Agreement:

www.tue.nl/taverne

Take down policy

If you believe that this document breaches copyright please contact us at:

openaccess@tue.nl

providing details and we will investigate your claim. 


\title{
On Packing, Connectivity, and Conductivity in Mesoscale Networks of Polydisperse Multiwalled Carbon Nanotubes
}

\author{
Karthikeyan Gnanasekaran, Gijsbertus de With,* and Heiner Friedrich \\ Laboratory of Materials and Interface Chemistry, Eindhoven University of Technology, Eindhoven, The Netherlands
}

Supporting Information

ABSTRACT: The formation of different mesoscale networks in multiwalled carbon nanotube (CNT) systems that are realized by the mixing of CNTs of varying lengths and length dispersities is reported. By this mixing process, we introduce competing length scales; hence, we alter the self-organized packing that contributes to the synergistic effects on the functional properties of the networks. The experimental findings show a gradual change of volume fraction and aspect ratio as well as a 2 -fold increase in electrical conductivity for such networks at certain specific compositions, hence an average aspect ratio. Quantitative large-area scanning transmission electron microscopy (STEM) imaging indeed revealed the existence of such mesoscale packing distribution variations. These packing observations suggest that these optimized networks of CNTs

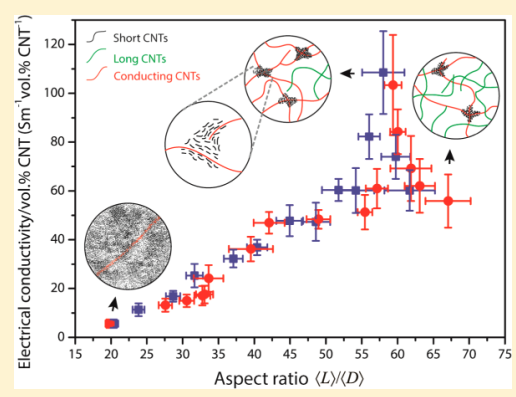
fit into an electrical conduction model that attributes its behavior to the formation of conduction knots, i.e., high-volume-fraction regions of relatively short CNTs that are connected by relatively long CNTs. If these conduction knots occupied by many short CNTs are distributed evenly with their nearest-neighbor distance being close to the average length of the CNT population, then the total electrical contact resistance in the conduction path will be effectively minimized. This study shows that optimized macroscale functional material properties can be designed into the initial colloidal dispersions by understanding and thus tuning the self-organization behavior of colloidal matter.

\section{INTRODUCTION}

Exploiting the potential of colloidal suspensions to self-organize into a variety of multiscale superstructures dependent on the size, shape, aspect ratio, or polydispersity of the sample population is an intriguing topic that has been investigated for many years but that is still not well understood. The length of particles (on the approximate scale of nanometers to millimeters) strongly influences the interactions and processes in which they may be involved, e.g., the stability of colloidal suspensions of nanoparticles is strongly influenced by the balance of repulsive and attractive forces. ${ }^{1}$ Carbon nanotubes (CNTs) are subjected to strong van der Waals and $\pi-\pi$ interactions that cause agglomeration and lead to the formation of inhomogeneous materials. Hence, the fabrication of CNTbased superstructures plays a vital role in translating the potential of CNTs into functional materials, such as CNTbased polymer nanocomposites, compacts, and thin films. ${ }^{2}$ It is the precise connectivity of each and every single CNT at the nanoscale that forms a mesoscale network, dictating the macroscopic functional properties. The relationship between macroscopic functional properties and nanoscale connectivity is still a matter of debate. It is widely accepted that CNTs connecting to other CNTs (forming a homogeneously distributed mesoscopic network) results in improved macroscopic properties such as electrical conductivity and mechanical stability. $^{3-5}$ Other studies, however, show that CNTs forming local agglomerates (introducing a secondary scale of length into the overall homogeneous CNT network) results in enhanced electrical conductivity. ${ }^{6-15}$ Nanoscale network assembly and connectivity are both governed by factors such as CNT shape, size, size distribution, straightness, flexibility, and attractive and repulsive interactions. ${ }^{16-18}$ The fundamental relationships among all these factors affect the resulting network topology; hence, the macroscopic properties are still largely unexplored. Simulations and theoretical predictions indicate the importance of polydispersity to electrical conductivity but only at the onset of network formation, i.e., at the percolation threshold. ${ }^{19-21}$ However, many technologically relevant applications, e.g., anodes or cathodes in electrochemical devices or catalytic supports, require the use of densely packed CNTs (in the form of porous compacts with volume fractions far above the percolation threshold). Dense packing of particles of different shapes (e.g., rods or spheres) at the macroscale was studied extensively, ${ }^{22-24}$ but such studies cannot be easily extrapolated to particles at the nanoscale because of the repulsive and attractive interactions that are predominant for nanomaterials. Currently, there are just a few experimental studies that address the electrical conductivity of CNT networks in the form of powders under compression. ${ }^{17,25,26}$ In these studies, the factors mentioned above (viz. shape, size, etc.) that govern electrical conductivity and network topology are scarcely investigated. Nevertheless, if CNTs are considered as a potentially realistic (raw) material for any upscaled and cost-effective production,

Received: August 12, 2014

Revised: November 17, 2014

Accepted: November 19, 2014

Published: November 19, 2014 
then these factors need attention because the raw-material CNTs most likely will need a broad distribution of values for these governing factors. In this article, we report on the influence of average CNT length (aspect ratio) and length distribution (polydispersity) on the packing and connectivity in porous compacts using three different CNT materials and their mixtures to derive a bottom-up multiscale model of CNT organization and macroscopic conductivity.

\section{MATERIALS AND METHODS}

Materials. Three CNT materials with different average lengths and polydispersities, furthermore referred to as $\mathrm{A}, \mathrm{B}$, and $\mathrm{C}$ (Chengdu Organic Chemicals Co. Ltd.) were chosen. All three raw materials were $95 \%$ pure and were synthesized by chemical vapor deposition. Representative TEM images of the raw materials are shown in Figure S1. All three materials consisted of nonstraight and polydisperse CNTs with size distributions skewed to shorter diameter and length. A quantitative analysis of the length and diameter distributions is presented in Figure S2. The weight-average lengths $\langle L\rangle$ of model materials $\mathrm{A}, \mathrm{B}$, and $\mathrm{C}$ were determined to be $377 \pm 12$, $1140 \pm 36$, and $1276 \pm 31 \mathrm{~nm}$ (mean \pm one standard deviation from the mean, using three independent measurements of $>200$ CNTs), respectively. The weight-average diameters $\langle D\rangle$ of model materials A, B, and C were almost identical $(18.9 \pm 0.2$, $19.0 \pm 0.3$, and $18.8 \pm 0.2 \mathrm{~nm}$, respectively), and all distributions had the same shape (Figure S2). CNT length was approximated by end-to-end distance, thus underestimating the actual length (Figure S3). The aspect ratio $\langle L\rangle /\langle D\rangle$ was calculated from the weight-average length divided by the diameter. Further details on the measurements of CNT size can be found in the Supporting Information, section III. CNT stock dispersions were prepared by adding $10 \mathrm{mg}$ of CNT material to $100 \mathrm{~mL}$ of $\mathrm{CHCl}_{3}$ (Biosolve, AR grade with purity = 99.9\%) and sonicating the mixture for $2 \mathrm{~h}$.

CNT Compact Preparation. For the preparation of a CNT compact, $10 \mathrm{~mL}$ of a CNT dispersion was vacuum filtered over a $0.2 \mu \mathrm{m}$ porous cellulose-mixed ester membrane filter (Whatman), as schematically illustrated in Figure 1. To vary the average aspect ratio and polydispersity of the CNTs in a compact, we mixed appropriate quantities of stock dispersions of two of the three raw materials. The mixed dispersions, referred to as $A B$ or $A C$, were further sonicated for an hour before vacuum filtration, resulting in $\mathrm{AB}$ - or $\mathrm{AC}$-type compacts. For example, $2 \mathrm{~mL}$ of model material A stock solution is mixed with $8 \mathrm{~mL}$ of model material $\mathrm{B}$ stock solution to form an $\mathrm{AB}$ type compact composed of $80 \%$ model material B and $20 \%$ model material A. The aspect ratio was calculated from the weight-average length divided by the weight-average diameter (see Supporting Information, section III for details).

Any residual solvent $\left(\mathrm{CHCl}_{3}\right)$ in the thus prepared compacts was removed by drying at room temperature for $24 \mathrm{~h}$. Some of the compacts were also heated to $70{ }^{\circ} \mathrm{C}$ for $24 \mathrm{~h}$ but did not show any differences in packing or conductivity compared to the room-temperature-dried compacts. Examples of CNT compacts are shown in Figure 1d.

Volume Fraction and Electrical Measurements. For each compact, the volume of the CNTs $V_{\mathrm{CNT}}$ was calculated from the known mass and density of the CNTs, whereas the volume of the compact $V_{\mathrm{COM}}$ was determined by measuring the physical dimensions of the compact. Bulk volume fractions $\varphi_{\mathrm{b}}$ of the prepared compacts were calculated as $V_{\mathrm{CNT}} / V_{\mathrm{COM}}$. Electrical measurements were carried out using a four-point

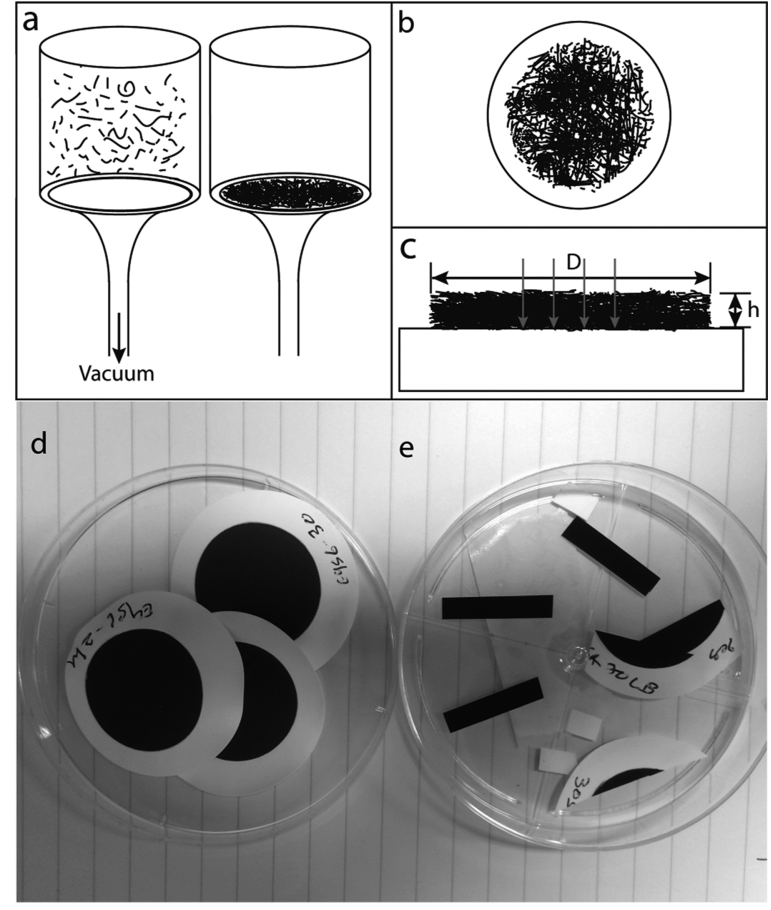

Figure 1. CNT compact preparation process: (a) schematic illustration of the CNT compact filtration process, (b) top view of CNT compacts, (c) side view of CNT compacts showing the thickness $h$ and the width $D$ of the compacts used for the volume fraction calculations, gray arrows illustrate four probes piercing through the thickness of the compacts to measure the electrical properties, (d) snapshot of CNT compacts as prepared, and (e) CNT compacts cut into strips for electrical measurements.

setup with parallel electrodes separated by $5 \mathrm{~mm}$, as schematically illustrated in Figure 1c. The current was applied through the outer electrodes by a Keithley 237 source measure unit, and the potential difference was measured between the inner electrodes by a Keithley 6517A electrometer. Detailed information on the calculation of $\varphi_{\mathrm{b}}$ and the measurement of electrical conductivity $\sigma$ is given in the Supporting Information, sections IV and V.

All other preparation and characterization details, such as electron microscopy sample preparation, imaging, and quantification by image analysis, can be found in the Supporting Information, sections VII-XVI.

\section{RESULTS AND DISCUSSION}

Global Packing. To analyze the overall variations in the packing density of compacts with respect to the variation of the aspect ratios, we calculated the bulk volume fraction $\varphi_{\mathrm{b}}$ and plotted the results as a function of the average aspect ratio in Figure 2a,b. For both AB- and AC-type compacts, $\varphi_{\mathrm{b}}$ approaches zero at high aspect ratios. To analyze quantitatively the randomly packed CNTs, we fitted the results to a widely accepted random contact model (RCM) derived from macroscopic data, ${ }^{27}$ as shown in Figure $2 \mathrm{c}$. The data points of our CNT compacts match closely with those of the macroscopic data where the volume fraction asymptotes toward the high-value aspect ratios. This indicates that $\varphi_{\mathrm{b}}$ scales with the measured average aspect ratio, despite the wide size distribution of the CNT population. Other studies show that the packing densities of polydisperse spheres are slightly higher than their monodisperse or bidisperse counterparts. ${ }^{28,29}$ In our 

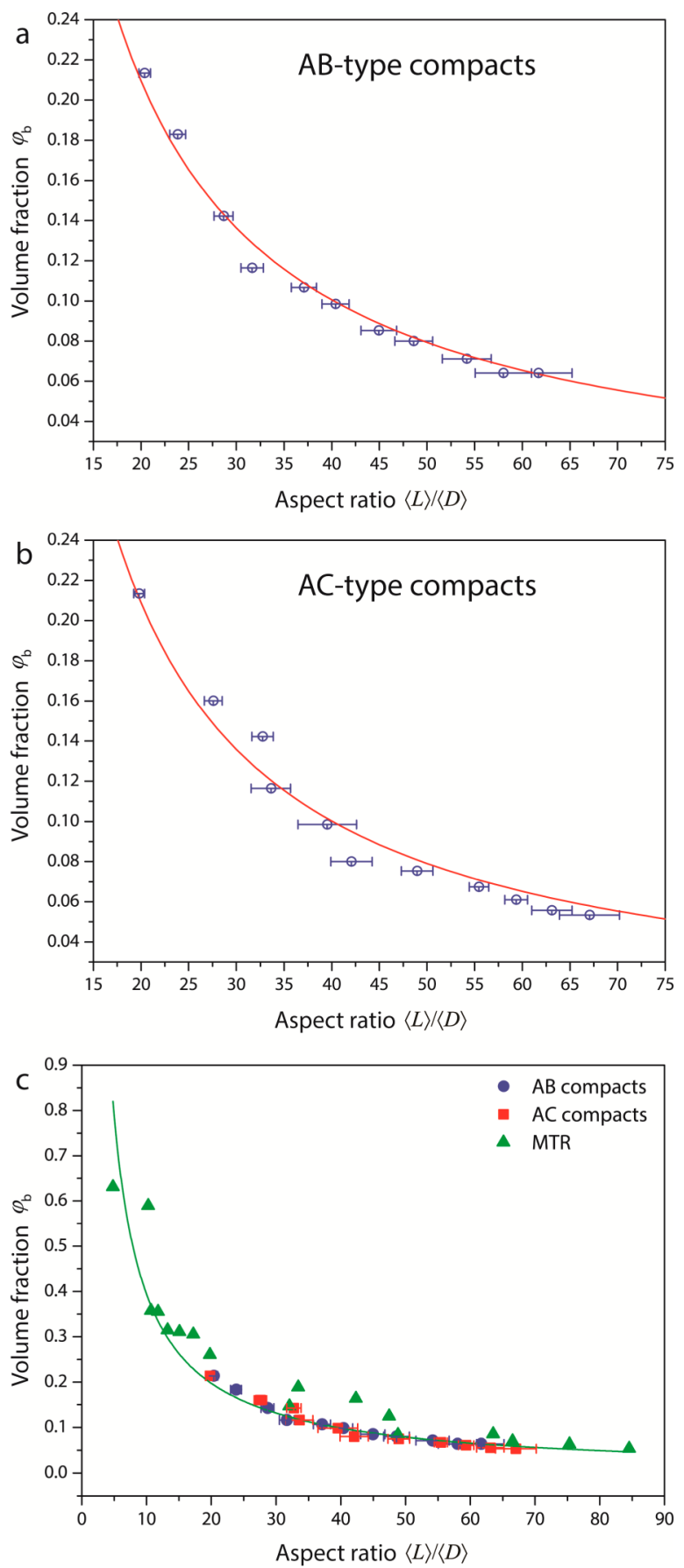

Figure 2. Measured bulk volume fraction $\varphi_{\mathrm{b}}$ plotted as a function of the average aspect ratio: (a) AB-type compacts, (b) AC-type compacts, and (c) data points of $\mathrm{AB}$ - and AC-type compacts overlaid with macroscopic data points, i.e., random packing of macroscopic thin rods (MTR). Green curve represents the best fit of the RCM function for the MTR data points taken from Philipse. ${ }^{27}$

system, the measured volume fractions of the compacts were at the lower end of the macroscopic data. We attribute this increased porosity to the nonstraightness of the CNTs (Figure S3), which has been shown to decrease the packing density. ${ }^{30}$

The RCM also predicts the dependence of the coordination number or average contact number per particle $\langle c\rangle$ on aspect ratio and volume fraction as expressed by

$$
\varphi \frac{\langle L\rangle}{\langle D\rangle} \approx \frac{\langle c\rangle}{2} \quad \text { for } \quad \frac{\langle L\rangle}{\langle D\rangle} \gg 1
$$

To confirm the RCM scaling predictions, we must independently measure $\varphi,\langle L\rangle /\langle D\rangle$, and $\langle c\rangle$. Unfortunately, experimental access to $\langle c\rangle$ is not feasible for our nanoscopic CNTs; hence, we approximated $\langle c\rangle$ by assuming that our CNT compacts follow the same trend as the RCM because the volume fraction and aspect ratio data points closely match the macroscopic data, as shown in Figure 2c. Although our data shows an excellent match with the RCM, we fitted our data points to $\varphi(L / D)^{\alpha}=\langle c\rangle / 2$ to compensate for the nonstraightness of the CNTs and the associated error involved in measurement of the CNT lengths. This yielded $\langle c\rangle \approx 10 \pm 2$, with $\alpha \approx 1.06 \pm 0.1$, for both $\mathrm{AB}$ - and AC-type compacts (see Supporting Information, section VI for more details on the calculation of $\langle c\rangle)$. RCM studies reported $\langle c\rangle \approx 6$ for aspect ratios close to one; $\langle c\rangle$ asymptotically increases to a constant value of $\sim 10$ for large aspect ratios. ${ }^{27,31}$ In comparison, studies on polydisperse and bidisperse spheres reported $\langle c\rangle$ values lower than those of monodisperse assemblies $(\langle c\rangle=6-4$, depending on the polydispersity). ${ }^{32,33}$ The value of $\alpha$ slightly larger than one and packing densities lower than those predicted by the RCM (Figure 2c) indeed indicate that the effective length of the CNTs is higher than the measured length (Figure S3), which in turn increases the effective excluded volume. The convolution of these nonstraightness effects is wrapped within the measurements of bulk volume fraction and aspect ratio but is detectable by the slight increase in $\alpha$. However, we believe that including more data points in the lower-value range of aspect ratios could give us a credible scaling constant. Because of the limitation of the size of the model materials, we cannot experimentally access the data points at low aspect ratios. Nevertheless, the overall random packing of nanoscopic CNTs follows the same trend as predicted by the macroscopic RCM, exhibiting a slight increase in $\alpha$.

Electrical Conduction. Figure 3a,b shows the measured electrical conductivity as a function of the volume fraction of the model materials and of the average aspect ratio of the model materials, respectively. The electrical conductivity is normalized by packing density and increases with increasing aspect ratio. This is due to a less disrupted flow of electrons along the conduction path, established by the long CNTs having fewer contact points. ${ }^{34,35}$ In addition to this general trend, it is also observed that the conductivity peaks at aspect ratios of between $\sim 55$ and $\sim 60$. This synergistic effect suggests that the electrical conductivity of the formed CNT networks does scale not only with volume fraction or aspect ratio but also depends strongly on other factors such as polydispersity, straightness, or attractive interactions that govern CNT network assembly and the resulting network topology over multiple length scales. To understand this behavior of electrical conductivity, we required knowledge of the distribution of the CNTs forming the physical network over multiple length scales.

Scanning Transmission Electron Microscopy (STEM) Imaging. As the synergistic effects in conductivity seem to be related to changes in network topology, we analyzed representative areas of the CNT network. Because such an area extends over tens of micrometers squared, a relatively large area needs to be analyzed. We approached this requirement by acquiring a large number of partially overlapping STEM images ( $\sim 100$ images $)$ with nanometer resolution, which were 

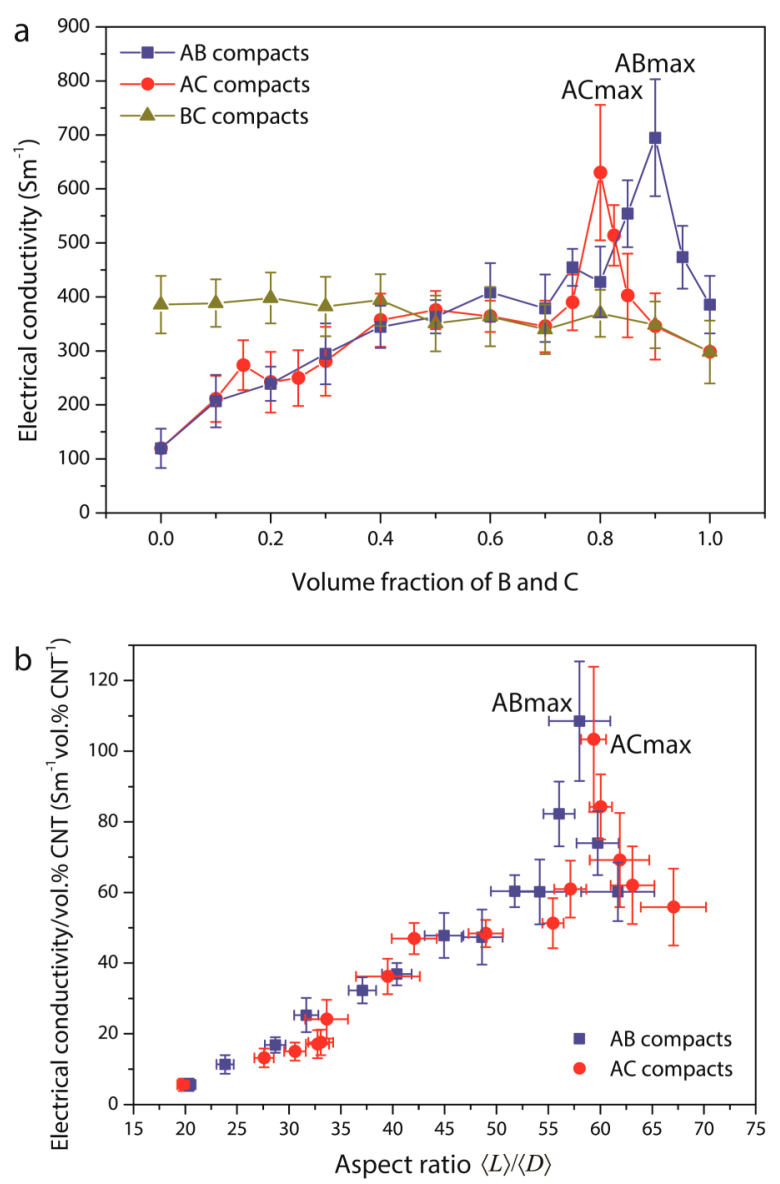

Figure 3. (a) Electrical conductivity plotted as a function of the relative volume fraction of model materials $\mathrm{B}$ and $\mathrm{C}$ with respect to model material A. (b) To exclude the effects of differences in packing density on the electrical conductivity curve, we normalized the conductivity values by the volume fraction and plotted these as a function of the average aspect ratio.

subsequently stitched together to form the required large area image for network analysis. To carry out the imaging, we opted for thick sections $(>500 \mathrm{~nm})$, which provides sufficient volume to be analyzed as well as optimizes the contrast and the resolution of the resultant STEM images. We carried out such large-area imaging, followed by quantitative analysis, for compacts $\mathrm{A}, \mathrm{B}, \mathrm{C}, \mathrm{AB}_{\max }$ and $\mathrm{AC}_{\max }$. More information on STEM sample preparation and imaging techniques can be found in the Supporting Information.

Representative morphologies of those large-area images are shown in Figure 4. Stitched high-resolution large-area images can also be found in Figure S5. Some clear differences are observed in the distribution of CNTs in the cross-section of the compacts. The images of compact A are the most homogeneous, indicating a very tightly packed CNT network without major variations in the mesoscale distribution of porosity. In contrast, significant mesoscopic variations exist in all of the other compacts, especially in $\mathrm{AB}_{\max }$ and $A C_{\max }$. The only difference between $\mathrm{B}$ and $\mathrm{AB}_{\max }$ (as between $\mathrm{C}$ and $\left.A C_{\text {max }}\right)$ is a slight increase in the number of shorter CNTs in the max-type compacts $\left(\mathrm{AB}_{\max }\right.$ and $\left.\mathrm{AC}_{\max }\right)$. This increase in shorter CNTs is responsible for the increase in densely packed agglomerates that can be seen as bright spots with diameters ranging between a few hundred nanometers and a few micrometers (Figure 4, arrows).

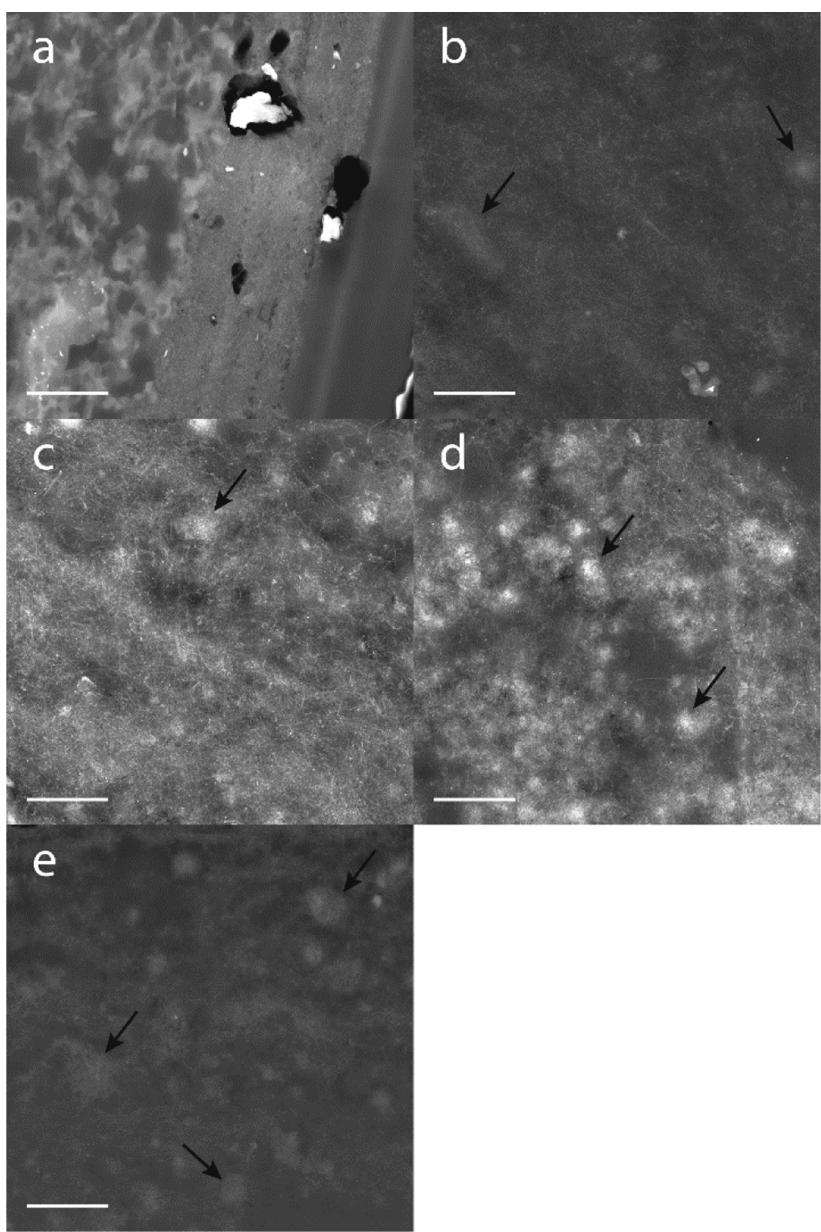

Figure 4. Representative HAADF-STEM images of CNT compacts: (a) compact A (showing the membrane filter paper and CNTs), (b) compact $\mathrm{B},(\mathrm{c})$ compact $\mathrm{C}$, (d) compact $\mathrm{AB}_{\max }$ and (e) compact $\mathrm{AC}_{\max }$. Scale bars represent a distance of $2 \mu \mathrm{m}$.

Formation of Mesoscopic Structures. The presence of these densely packed mesoscopic regions can be explained as follows. Using a continuum model, Girifalco et al. derived an intermolecular interaction potential between two individual CNTs in vacuum. ${ }^{36}$ A large attractive interaction is generated between individual CNTs where the attraction between the particles is at least 40 times the thermal energy $\mathrm{kT}$, i.e., $>40 \mathrm{kT} /$ $\mathrm{nm} .^{37}$ In our experiments involving the vacuum filtration of CNT dispersions, similar interactions are present. Additionally, the solvent $\left(\mathrm{CHCl}_{3}\right)$ as well as the employed CNT concentration plays an important role in the colloidal stability of the dispersion. A complete understanding of the effect of solvent on the interactions between CNTs is lacking at present. We minimized the effect of solvent on the randomness of the dispersion by using a stable dispersion and immediately vacuum filtered after sonication. Hence, any intermolecular interactions leading to structure formation in the dispersion occurred only during the postsonication, filtration, and drying times (maximum $\sim 30 \mathrm{~s}$ ). Within this limited time frame, a unique network topology arises mainly as a result of the interaction of the polydisperse CNTs and their rapid jamming together during filtration. Although we tried to prevent as far as possible the influence of the solvent on the process prior to the formation of the CNT compacts, the effects of the solvent on the final compact morphology cannot be excluded. However, 
the stability of the CNT dispersion in chloroform indicates that significant aggregation in the dispersed state is unlikely. More detailed discussions of the stability of the solvent effects and their analyses can be found in Supporting Information, section $X$. During the filtration process, the concentration of the colloidal dispersion mixture is quickly increased to a maximum concentration. In the beginning of the process, the longer CNTs are jammed together and form the somewhat open skeleton of the compact. This predominantly controls the global packing density. Meanwhile, short CNTs are caged in the open pores of the jammed, longer CNTs. As the concentration of the colloidal dispersion increases, the shorter $\mathrm{CNTs}$ are subsequently caught in the open pores and attached to the nearby jammed-together longer CNTs, contributing to the local packing density. The network topology of such a system is schematically illustrated in Figure 5. However, not all of the CNTs contribute to the formation of such mesoscopic structures. In some images, a thin layer of a uniformly distributed and tightly packed CNT network is noticed close to the membrane filter; we believe that this is mainly due to the deposition of shorter CNTs located at the bottom of the colloidal CNT suspension that immediately formed a layer upon the application of vacuum (Figure S5e). We believe that these densely packed mesoscopic structures can be related to the synergistic effect on conductivity because studies show the enhanced charge-transfer properties of CNT-rich regions (densely packed regions). ${ }^{6,7,12,13,15}$ To understand and derive a bottom-up model of this behavior, the frequency and distribution of these dense regions in the compacts need to be quantitatively analyzed.

Quantification of Large-Area STEM Images. The quantification process was carried out on compacts that showed mesoscale variations, namely, $\mathrm{B}, \mathrm{AB}_{\max }, \mathrm{C}$, and $\mathrm{AC}_{\max }$. In brief, the quantification process consists of (1) removal of noise by median filtering, (2) correction for background intensity, (3) removal of regions with catalyst debris, (4) creation of a calibration plot, i.e., intensity versus CNT packing density, (5) classification on the basis of packing density, and (6) statistical analysis of the neighbor distances between densely packed regions. A detailed description of the quantification process is given in the Supporting Information, section XI. On the basis of the local volume fraction $\varphi$ of the CNTs and the bulk volume fraction $\varphi_{\mathrm{b}}$ of a particular compact, as calculated from experimental data, we classified the packing density throughout each compact in such a way that regions where $\varphi<\varphi_{\mathrm{b}}$ are set as 0 and regions where $\varphi>\varphi_{\mathrm{b}}$ are set as 1 , as shown in Figure 6. The respective area fractions for all compacts are shown in Table 1. The total area of densely packed regions in the max-type compacts was higher than those of B- and C-type compacts. It is difficult to correlate these data to the relative change in the size and amount of CNTs so we might derive a criterion for the change in topology; hence, it is also difficult to derive such a criterion for the electrical conductivity. However, we can surmise that the synergistic effect on conductivity is related to the dense regions and their distribution; hence, we decided to correlate the effects of conductivity to the difference in high-volume-fraction regions of the compacts $\left(\varphi_{\mathrm{h}}=\varphi>\varphi_{\mathrm{b}}\right.$; Figure $6 \mathrm{~b}$, white regions).

The histogram of CNT-rich regions (Figure S10) shows that a greater number of dense regions exist in max-type compacts than in B- or C-type compacts. The center-center nearestneighbor and second-nearest-neighbor distances of CNT-rich regions $\left(\varphi>\varphi_{\mathrm{b}}\right)$ in compacts $\mathrm{B}, \mathrm{C}, \mathrm{AB}_{\max }$ and $\mathrm{AC}_{\max }$ were also

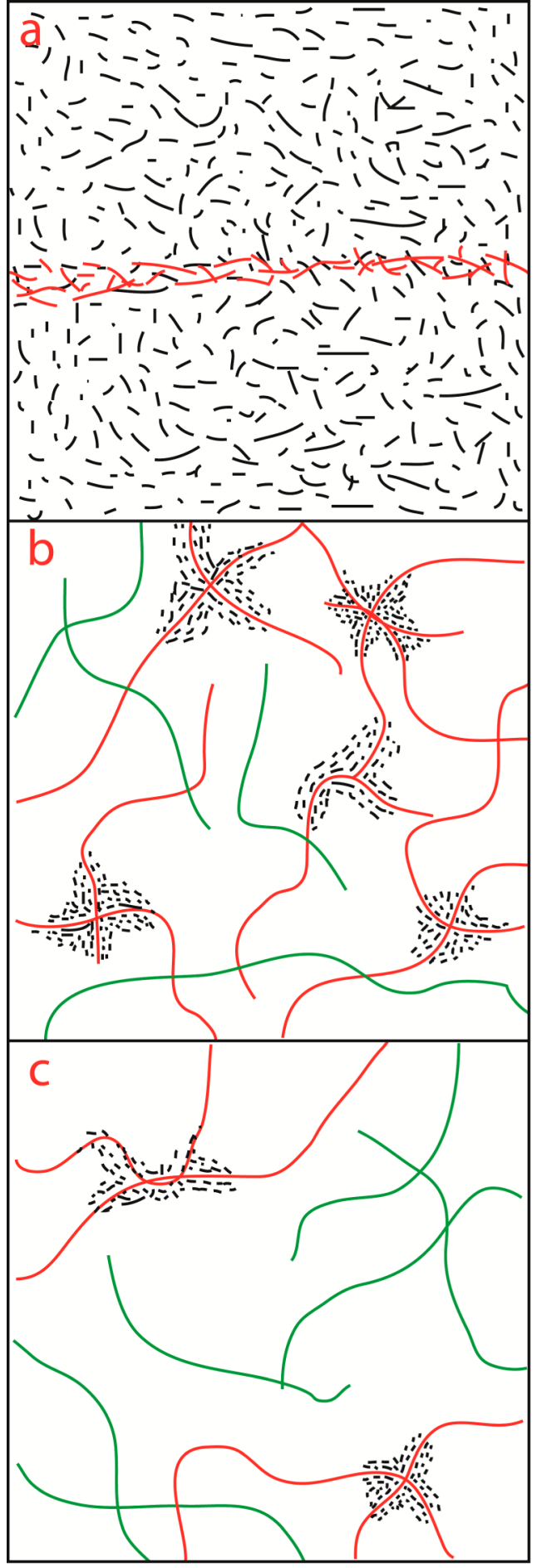

Figure 5. Schematic illustration of the network topology of various compacts: (a) compact $\mathrm{A},(\mathrm{b})$ compact $\mathrm{AB}_{\max }$ or $\mathrm{AC}_{\max }$ (c) compact $\mathrm{B}$ or C. Black lines are short CNTs, green lines are long CNTs, and red lines indicate the electron conduction pathway across the network.

measured. For the max-type compacts, an almost 2-fold decrease is noticed in the nearest-neighbor distance $D_{1 \mathrm{nn}}$ and the second-nearest-neighbor distance $D_{2 \mathrm{nn}}$ compared with those of B- and C-type compacts (Table 2). The distribution of neighbor distances in the max-type compacts and B- and C-type compacts can be found in Figure S11.

Electrical Conduction Model. On the basis of the ratio between neighbor distances of high-volume-fraction regions $\varphi_{\mathrm{h}}$ 


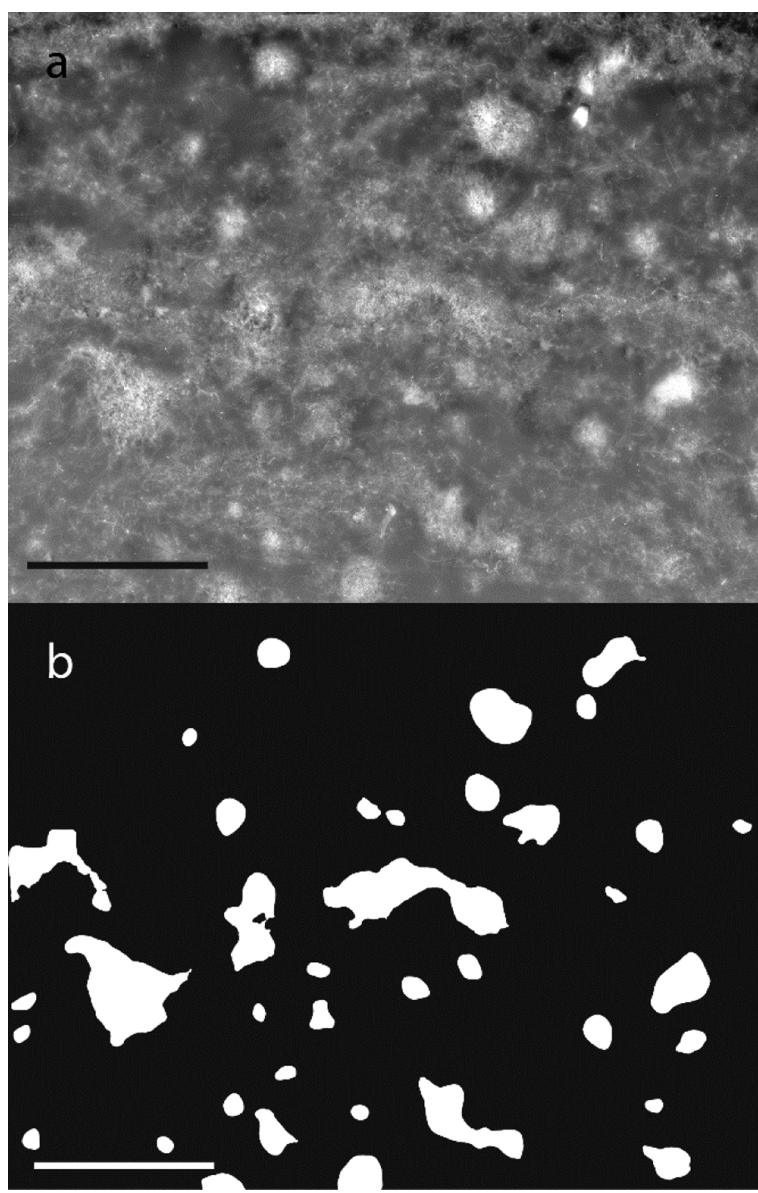

Figure 6. (a) HAADF-STEM image of compact $\mathrm{AC}_{\max }$ illustrating the distribution in the volume fraction. (b) Segmentation of the network topology of compact $\mathrm{AC}_{\max }$ based on the measured bulk volume fraction $\varphi_{\mathrm{b}}$. Black, $\varphi_{\mathrm{l}}=\varphi<\varphi_{\mathrm{b}}$; white, $\varphi_{\mathrm{h}}=\varphi>\varphi_{\mathrm{b}}$. Scale bars represent a distance of $3 \mu \mathrm{m}$.

and the corresponding weight-average length of CNTs, an electrical conduction model can be formulated. As mentioned above, previous studies have revealed the enhanced chargetransfer properties of CNT-rich networks, i.e., high-volumefraction regions, because of the increase in the effective CNTCNT contact area. ${ }^{6,7,12,13,15}$ (More detailed discussion on electrical conduction mechanism and structural implications is given in the Supporting Information, section XIV.) To obtain enhanced macroscopic conductivity across the compacts, these high-volume-fraction regions have to be connected by lowresistance pathways. A low-resistance pathway connecting two high-volume-fraction regions is obtained when the connecting pathways possess an as-small-as-possible resistance, i.e., the connecting pathways have the least possible number of contacts; this can be effectively achieved by a single long $\mathrm{CNT}$, as is likely to occur in compacts $\mathrm{AB}_{\text {max }}$ and $\mathrm{AC}_{\max }$.

In compacts $\mathrm{B}$ and $\mathrm{C}$, the average nearest-neighbor distance is substantially greater than the average length $L$ of CNTs, i.e.,
Table 2. Center-Center Nearest-Neighbor Distance and Second-Nearest-Neighbor Distance of High-VolumeFraction Regions $\left(\varphi>\varphi_{\mathrm{b}}\right)$ in Various CNT Compacts

\begin{tabular}{lrll} 
& avg length $\langle L\rangle(\mathrm{nm})$ & \multicolumn{1}{c}{$D_{1 \mathrm{nn}}(\mathrm{nm})$} & \multicolumn{1}{c}{$D_{2 \mathrm{nn}}(\mathrm{nm})$} \\
$\mathrm{A}$ & $377 \pm 12$ & & \\
$\mathrm{~B}$ & $1140 \pm 36$ & $1894 \pm 182$ & $2866 \pm 221$ \\
$\mathrm{C}$ & $1276 \pm 31$ & $1962 \pm 153$ & $2780 \pm 249$ \\
$\mathrm{AB}_{\max }$ & $1072 \pm 55$ & $1137 \pm 78$ & $1835 \pm 148$ \\
$\mathrm{AC}_{\max }$ & $1130 \pm 23$ & $1305 \pm 121$ & $1784 \pm 156$ \\
\hline
\end{tabular}

$D_{1 \mathrm{nn}} \approx 1.5 L$ and $D_{2 \mathrm{nn}} \gtrsim 2 L$. Hence, there is a much lower probability that these high-volume-fraction regions can be connected by single CNTs throughout the entire network. In contrast, if the nearest-neighbor distance approaches the average length, then there is a higher probability of connecting high-volume-fraction regions by single CNTs, leading to lowresistance charge-transfer units connecting several concentrated sites that form an effective pathway for electron conduction. The fact that $D_{1 \mathrm{nn}}$ approaches $L$ and $D_{2 \mathrm{nn}} \lesssim 2 L$ for compacts $\mathrm{AB}_{\max }$ and $\mathrm{AC}_{\max }$ is suggestive of such a phenomenon possibly occuring in max-type compacts. Nonetheless, the 2D-analysis information is averaged over the entire thickness, which underestimates the actual $3 \mathrm{D}$ distances by up to $10 \%$ of $D_{1 \mathrm{nn}}$ and by up to $4 \%$ of $D_{2 n n}$ in the max-type compacts (see Supporting Information, section $\mathrm{XV}$, for more information on this estimation). Even taking this additional uncertainty into account, the difference in nearest-neighbor distance between compacts $\mathrm{B}$ and $\mathrm{AB}_{\max }$ or between compacts $\mathrm{C}$ and $\mathrm{AC}_{\max }$ is still significant.

Now, looking at the compacts with low aspect ratios, such as compact $\mathrm{A}$, charge transfer is hindered by a huge number of contact points that contribute to a high contact resistance. We analyzed the contact properties of CNTs in the compacts by preparing composites of compacts via a polymer infiltration (PI) process driven by capillary forces (see Supporting Information, section XVI for more information). Because of the inclusion of a polymer matrix, the contact resistances at contact points in the conduction pathway increased, as shown in Figure 7. In addition to the increased resistance, we also observed that the relative increase is larger for the low-aspectratio compacts. This indeed implies the involvement of a large number of contact points at low aspect ratios, where the electron flow is hindered mainly by the number of contact points on the path. Because the minimum resistance is still observed in polymer-infiltrated compacts $\mathrm{AB}_{\text {max }}$ and $\mathrm{AC}_{\text {max }}$, the presented structural model, i.e., dense regions connected by few long CNTs, still applies. Furthermore, it can be concluded that polymer infiltration increases the contact resistance between CNTs but does not disentangle the initially formed network so that the functional properties are maintained.

\section{CONCLUSIONS}

We have shown that changing the average length and length distribution of CNTs can influence the local packing although

Table 1. Average Area Occupied by Three Different Packing Distributions in Various CNT Compacts ${ }^{a}$

$\begin{array}{crrr}\varphi & \text { compact B } & \text { compact C } & \text { compact } \mathrm{AB}_{\max } \\ \varphi_{1}=\varphi<\varphi_{\mathrm{b}} & 93.30 \pm 3.27 & 95.32 \pm 1.94 & 91.30 \pm 2.86 \\ \varphi_{\mathrm{h}}=\varphi>\varphi_{\mathrm{b}} & 6.69 \pm 2.27 & 4.67 \pm 1.29 & 8.68 \pm 1.12\end{array}$

${ }^{a}$ Represented in percent. 

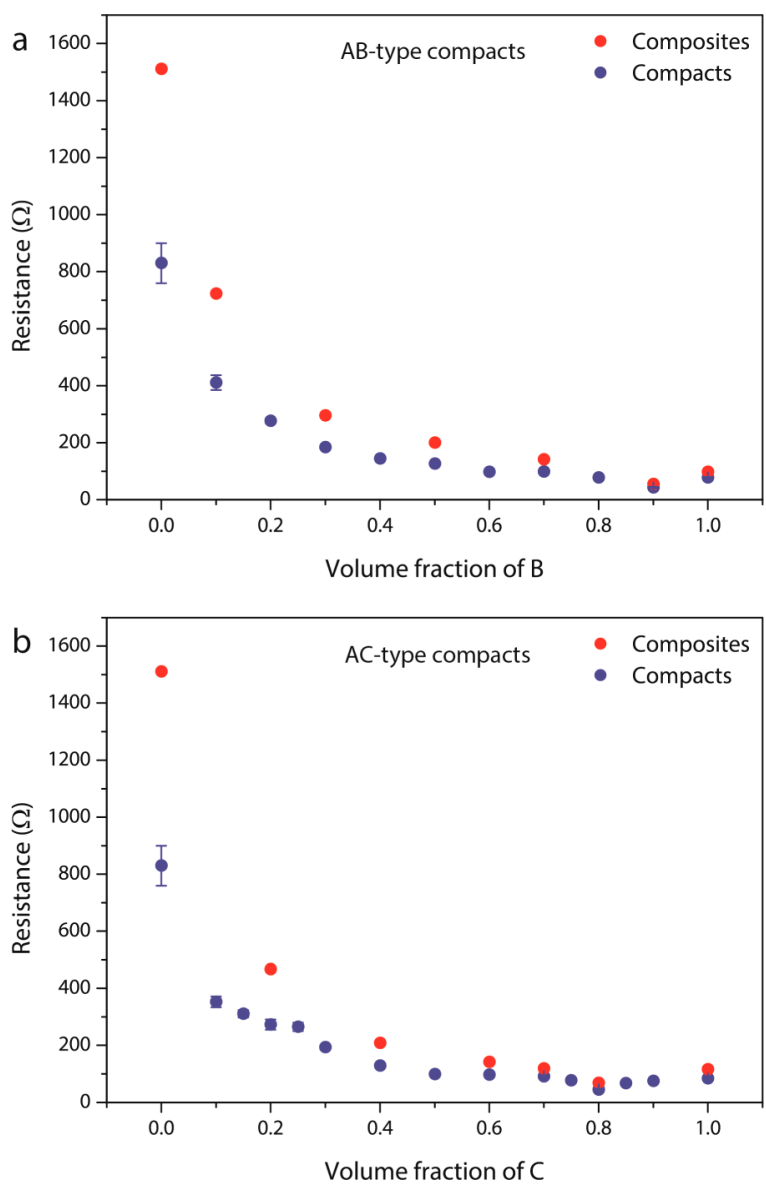

Figure 7. Measured resistance of various CNT compacts plotted as a function of the relative volume fraction of model materials: (a) ABtype compacts and (b) AC-type compacts.

the global packing follows trends similar to those observed in the packing of macroscopic rod-shaped particles. The comparison of nanoscopic data with macroscopic data reveals a surprising continuity of global packing phenomena for 6-9 orders of magnitude of length scales, irrespective of the size distribution. However, as a result of strong attraction among CNTs of varying length and polydispersity, the functional properties, such as the electrical conductivity, can be influenced. We analyzed a representative volume of CNT networks by a combination of large-area imaging and image quantification to develop a bottom-up understanding of the effect of polydispersity on electrical conductivity. Polydisperse systems assemble on the mesoscale so that the longest CNTs create a jammed-together porous compact in which dense regions of shorter CNTs can be found. On optimizing this morphology so that CNT-rich regions (high-volume-fraction regions) are distributed evenly with a nearest-neighbor distance close to the average length of the CNTs, the total electrical contact resistance can be minimized. Preserving the initially formed network by ex situ infiltration of a polymer matrix can conveniently accommodate the fabrication of functional devices.

The current study can be extended by controlling the fabrication process of CNT compacts. However, to derive a definitive understanding of this multiscale behavior, factors such as CNT polydispersity, straightness, flexibility, and attractive and repulsive interactions have to be studied in parallel. In this way, a predictive capability can be developed for the development of controlled functional properties. Additional progress in $3 \mathrm{D}$ nanoscale imaging and image analysis, such as the measurement of real contact points, would push our understanding further. Nevertheless, the current findings add to the fundamental understanding of the packing of rodlike nanofillers or nanocolloids with different aspect ratios and the modeling of macroscopic electrical conductivity using mesoscopic properties such as network topology.

\section{ASSOCIATED CONTENT}

\section{S Supporting Information}

TEM images, size distribution and size measurement details of the CNT raw materials, experimental details and schematics, STEM imaging techniques, large-area images, discussions of solvents (UV absorption analysis), quantification methodology, nearest-neighbor distances and size distribution of highvolume-fraction regions of the CNT network, and details of the electrical conduction mechanism. This material is available free of charge via the Internet at http://pubs.acs.org.

\section{AUTHOR INFORMATION}

\section{Corresponding Author}

*E-mail: G.deWith@tue.nl.

\section{Notes}

The authors declare no competing financial interest.

\section{ACKNOWLEDGMENTS}

We received funding from the European Union Seventh Framework Programme (FP7-MC-ITN) under grant agreement no. 264710. We thank the Directorate-General for Science, Research, and Development of the European Commission for financial support. We thank Prof. A. Philipse and Prof. P. van der Schoot for insightful discussions and suggestions.

\section{REFERENCES}

(1) Talapin, D. V. LEGO Materials. ACS Nano 2008, 2, 1097-1100.

(2) Shimizu, M.; Fujii, S.; Tanaka, T.; Kataura, H. Effects of Surfactants on the Electronic Transport Properties of Thin-Film Transistors of Single-Wall Carbon Nanotubes. J. Phys. Chem. C 2013, 117, 11744-11749.

(3) Song, Y. S.; Youn, J. R. Influence of Dispersion States of Carbon Nanotubes on Physical Properties of Epoxy Nanocomposites. Carbon 2005, 43, 1378-1385.

(4) Xie, X.-L.; Mai, Y.-W.; Zhou, X.-P. Dispersion and Alignment of Carbon Nanotubes in Polymer Matrix: A Review. Mater. Sci. Eng., $R$ 2005, 49, 89-112.

(5) Du, F.; Scogna, R. C.; Zhou, W.; Brand, S.; Fischer, J. E.; Winey, K. I. Nanotube Networks in Polymer Nanocomposites: Rheology and Electrical Conductivity. Macromolecules 2004, 37, 9048-9055.

(6) Cardoso, P.; Silva, J.; Klosterman, D.; Covas, J.; van Hattum, F.; Simoes, R; Lanceros-Mendez, S. The Influence of the Dispersion Method on the Electrical Properties of Vapor-Grown Carbon Nanofiber/Epoxy Composites. Nanoscale Res. Lett. 2011, 6, 370.

(7) Aguilar, J. O.; Bautista-Quijano, J. R.; Aviles, F. Influence of Carbon Nanotube Clustering on the Electrical Conductivity of Polymer Composite Films. eXPRESS Polym. Lett. 2010, 4, 292-299.

(8) Bryning, M. B.; Islam, M. F.; Kikkawa, J. M.; Yodh, A. G. Very Low Conductivity Threshold in Bulk Isotropic Single-Walled Carbon Nanotube-Epoxy Composites. Adv. Mater. 2005, 17, 1186-1191.

(9) Martin, C. A.; Sandler, J. K. W.; Shaffer, M. S. P.; Schwarz, M. K.; Bauhofer, W.; Schulte, K.; Windle, A. H. Formation of Percolating Networks in Multi-Wall Carbon-Nanotube-Epoxy Composites. Compos. Sci. Technol. 2004, 64, 2309-2316. 
(10) Du, F.; Guthy, C.; Kashiwagi, T.; Fischer, J. E.; Winey, K. I. An Infiltration Method for Preparing Single-Wall Nanotube/Epoxy Composites with Improved Thermal Conductivity. J. Polym. Sci., Part B: Polym. Phys. 2006, 44, 1513-1519.

(11) Nogales, A.; Broza, G.; Roslaniec, Z.; Schulte, K.; Šics, I.; Hsiao, B. S.; Sanz, A.; García-Gutiérrez, M. C.; Rueda, D. R.; Domingo, C.; et al. Low Percolation Threshold in Nanocomposites Based on Oxidized Single Wall Carbon Nanotubes and Poly(butylene terephthalate). Macromolecules 2004, 37, 7669-7672.

(12) Sandler, J. K. W.; Kirk, J. E.; Kinloch, I. A.; Shaffer, M. S. P.; Windle, A. H. Ultra-Low Electrical Percolation Threshold in CarbonNanotube-Epoxy Composites. Polymer 2003, 44, 5893-5899.

(13) Pegel, S.; Pötschke, P.; Petzold, G.; Alig, I.; Dudkin, S. M.; Lellinger, D. Dispersion, Agglomeration, and Network Formation of Multiwalled Carbon Nanotubes in Polycarbonate Melts. Polymer 2008, 49, 974-984.

(14) Alig, I.; Skipa, T.; Engel, M.; Lellinger, D.; Pegel, S.; Pötschke, P. Electrical Conductivity Recovery in Carbon Nanotube-Polymer Composites After Transient Shear. Phys. Status Solidi B 2007, 244, $4223-4226$.

(15) Li, J.; Ma, P. C.; Chow, W. S.; To, C. K.; Tang, B. Z.; Kim, J. K. Correlations Between Percolation Threshold, Dispersion State, and Aspect Ratio of Carbon Nanotubes. Adv. Funct. Mater. 2007, 17, 3207-3215.

(16) Kyrylyuk, A. V.; Hermant, M. C.; Schilling, T.; Klumperman, B.; Koning, C. E.; van der Schoot, P. Controlling Electrical Percolation in Multicomponent Carbon Nanotube Dispersions. Nat. Nanotechnol. 2011, 6, 364-369.

(17) Shui, X.; Chung, D. D. L. Electrical Resistivity of SubmicronDiameter Carbon-Filament Compacts. Carbon 2001, 39, 1717-1722.

(18) Hermant, M. C.; Klumperman, B.; Kyrylyuk, A. V.; van der Schoot, P.; Koning, C. E. Lowering the Percolation Threshold of Single-Walled Carbon Nanotubes using Polystyrene/Poly(3,4-Ethylenedioxythiophene): Poly(Styrene Sulfonate) Blends. Soft Matter 2009, 5, 878-885.

(19) Otten, R. H. J.; van der Schoot, P. Continuum Percolation of Polydisperse Nanofillers. Phys. Rev. Lett. 2009, 103, 225704.

(20) Otten, R. H. J.; van der Schoot, P. Connectivity Percolation of Polydisperse Anisotropic Nanofillers. J. Chem. Phys. 2011, 134, 094902.

(21) Kyrylyuk, A. V.; van der Schoot, P. Continuum Percolation of Carbon Nanotubes in Polymeric and Colloidal Media. Proc. Natl. Acad. Sci. U.S.A. 2008, 105, 8221-8226.

(22) Wouterse, A.; Luding, S.; Philipse, A. P. On Contact Numbers in Random Rod Packings. Granular Matter 2009, 11, 169-177.

(23) Zhao, J.; Li, S.; Zou, R.; Yu, A. Dense Random Packings of Spherocylinders. Soft Matter 2012, 8, 1003-1009.

(24) Farr, R. S.; Groot, R. D. Close Packing Density of Polydisperse Hard Spheres. J. Chem. Phys. 2009, 131, 244104.

(25) Shaffer, M. S. P.; Fan, X.; Windle, A. H. Dispersion and Packing of Carbon Nanotubes. Carbon 1998, 36, 1603-1612.

(26) Marinho, B.; Ghislandi, M.; Tkalya, E.; Koning, C. E.; de With, G. Electrical Conductivity of Compacts of Graphene, Multi-Wall Carbon Nanotubes, Carbon Black, and Graphite Powder. Powder Technol. 2012, 221, 351-358.

(27) Philipse, A. P. The Random Contact Equation and Its Implications for (Colloidal) Rods in Packings, Suspensions, and Anisotropic Powders. Langmuir 1996, 12, 1127-1133.

(28) Ogarko, V.; Luding, S. Equation of State and Jamming Density for Equivalent Bi- and Polydisperse, Smooth, Hard Sphere Systems. J. Chem. Phys. 2012, 136, 124508.

(29) Rosenberg, R. T.; Dan, N. Effect of Colloidal Particle Size on Adsorbed Monodisperse and Bidisperse Monolayers. Langmuir 2011, 27, 8729-8734.

(30) Gravish, N.; Franklin, S. V.; Hu, D. L.; Goldman, D. I. Entangled Granular Media. Phys. Rev. Lett. 2012, 108, 208001.

(31) Blouwolff, J.; Fraden, S. The Coordination Number of Granular Cylinders. Europhys. Lett. 2006, 76, 1095-1101.
(32) Kristiansen, K. D. L.; Wouterse, A.; Philipse, A. Simulation of Random Packing of Binary Sphere Mixtures by Mechanical Contraction. Physica A 2005, 358, 249-262.

(33) Wiącek, J.; Molenda, M. Effect of Particle Polydispersity on Micromechanical Properties and Energy Dissipation in Granular Mixtures. Particuology 2014, 16, 91-99.

(34) Shehzad, K.; Dang, Z.-M.; Ahmad, M. N.; Sagar, R. U. R.; Butt, S.; Farooq, M. U.; Wang, T.-B. Effects of Carbon Nanotubes Aspect Ratio on the Qualitative and Quantitative Aspects of Frequency Response of Electrical Conductivity and Dielectric Permittivity in the Carbon Nanotube/Polymer Composites. Carbon 2013, 54, 105-112.

(35) Lisunova, M. O.; Mamunya, Y. P.; Lebovka, N. I.; Melezhyk, A. V. Percolation Behaviour of Ultrahigh Molecular Weight Polyethylene/Multi-Walled Carbon Nanotubes Composites. Eur. Polym. J. 2007, 43, 949-958.

(36) Girifalco, L. A.; Hodak, M.; Lee, R. S. Carbon Nanotubes, Buckyballs, Ropes, and A Universal Graphitic Potential. Phys. Rev. B 2000, 62, 13104-13110.

(37) Szleifer, I.; Yerushalmi-Rozen, R. Polymers and Carbon Nanotubes-Dimensionality, Interactions and Nanotechnology. Polymer 2005, 46, 7803-7818. 